\title{
Identification and validation of potential novel prognostic biomarkers for patients with glioma based on a gene co-expression network
}

\author{
Yan-Wei Jiang, Rui Wang, Yuan-Dong Zhuang, Chun-Mei Chen \\ Department of Neurosurgery, Fujian Medical University Union Hospital, Fuzhou, China \\ Contributions: (I) Conception and design: YW Jiang, CM Chen; (II) Administrative support: YW Jiang; (III) Provision of study materials or patients: \\ YW Jiang, R Wang, YD Zhuang; (IV) Collection and assembly of data: YW Jiang, R Wang, YD Zhuang; (V) Data analysis and interpretation: All \\ authors; (VI) Manuscript writing: All authors; (VII) Final approval of manuscript: All authors. \\ Correspondence to: Yan-Wei Jiang. Department of Neurosurgery, Fujian Medical University Union Hospital, No. 29 Xinquan Road, Gulou, Fuzhou, \\ China. Email: joway12@126.com.
}

Background: Glioma is widely regarded as one of most lethal and challenging diseases of the nervous
system. The aim of this study was to identify novel biomarkers that offer better prognosis prediction for
Chinese patients with glioma.

Methods: By using systematic approaches, the co-expression modules were identified from the Chinese Glioma Genome Atlas (CGGA) database through weighted gene co-expression network analysis and functional enrichment of essential modules of Kyoto Encyclopedia of Genes and Genomes terms. The co-expression modules were validated using The Cancer Genome Atlas database and the protein-protein interaction (PPI) network.

Results: For network construction, 5,374 among 21,494 genes were selected, and an increasing genetic variance was associated with the prognosis of glioma. By using functional enrichment analysis, the involvement of multiple vital processes, including metabolism of fatty acids, was correlated with the patient prognosis. Notably, five hub genes (KCNB1, UST, SOX8, KLHL42, and HDAC4) were identified for these processes. Accordingly, using the Kaplan-Meier method, there was enhanced expression of these genes in patients with significantly lower overall survival rates, especially those from the CGGA database.

Conclusions: This study not only revealed the essential co-expression gene modules in patients with glioma, but it also unraveled the potential signaling pathways underlying these functional processes.

Keywords: The Chinese Glioma Genome Atlas (CGGA); glioma; gene co-expression network; weighted gene coexpression network analysis (WGCNA); hub genes

Submitted Jan 12, 2020. Accepted for publication Aug 28, 2020.

doi: $10.21037 /$ tcr-20-492

View this article at: http://dx.doi.org/10.21037/tcr-20-492

\section{Introduction}

Glioma, whose prevalence varies substantially with patient age, gender, race, and a number of other clinicopathological parameters, is widely regarded as one of most lethal and challenging diseases of the nervous system; the median survival time of glioma patients is $5-10$ years, and the estimated 10 -year survival rate varies between $5 \%$ and $50 \%$ $(1,2)$. Moreover, following a tumorectomy, the combined use of both concurrent radiation and temozolomide treatment is currently the standard therapy for patients with glioma. Notably, even though gliomas are generally subdivided into two subgroups based on a known profile of related biomarkers (3), i.e., low-grade gliomas and high-grade gliomas, patients with the same diagnostic results sometimes have totally different outcomes even after the same therapeutic procedures. For instance, 
after approximately 6.9 months, patients with high-grade gliomas are more likely to have recurrent glioma, resulting in a median survival time of about $12-15$ months in these patients following diagnosis (2). Therefore, despite surgical success, the clinical outcome of glioma patients is generally unsatisfactory (4).

Many studies have tried to find a therapeutic target or prognostic biomarker for glioma to improve clinical outcomes $(5,6)$. Mutations in the genes isocitrate dehydrogenase (IDH) 1 and 2 have been reported in $73-85 \%$ of secondary glioblastomas and $72-100 \%$ of stage II and III astrocytic and oligodendroglial tumors, but these mutations are almost never found in primary glioblastomas $(5,6)$. IDH mutations represent a major biomarker with diagnostic, prognostic, and predictive implications (6). In addition, promoter methylation of O6-methylguanineDNA methyltransferase (MGMT) is found in about $80 \%$ of low-grade IDH-mutated gliomas. Low MGMT levels correlate with modestly improved survival (5). Moreover, the epidermal growth factor receptor (EGFR) is amplified in about $40 \%$ of glioblastoma patients, and it is often associated with high-grade classical tumors. However, whether EGFR amplification can be used as a prognostic biomarker is controversial $(5,6)$. Recently, the Rho-specific guanine-nucleotide exchange factor PLEKHG5 was reported to be involved in glioma migration, invasion, and epithelial-mesenchymal transition and could be used as a prognostic biomarker for glioma patients (7). Furthermore, some other signaling pathways including the p53 pathway, phosphoinositide 3-kinase pathway, retinoblastoma pathway, and Ras/Raf/mitogen-activated protein kinase (MAPK) pathway also have been reported to be involved in the progression of glioma $(5,6)$. However, these studies may have overemphasized the determination of a differential expression profile while ignoring the crosstalk between genes and/or signaling pathways, which are likely to be functionally correlated. Therefore, the clinical application of these biomarkers is limited.

During the last decade, studies have started to focus on unscrambling related gene expression profiles, leading to a better understanding of the potential molecular mechanisms underlying the formation and progression of glioma. By using weighted gene co-expression network analysis (WGCNA) based on The Cancer Genome Atlas (TCGA) database, $\mathrm{Xu}$ et al. have found that gene prognosis models with four genes (OSMR + SOX21 + MED10 + PTPRN) can be used for estimating overall survival in glioblastoma patients (8). However, since samples in the TCGA database are mainly from the USA, some conclusions drawn from the TCGA database may not be suitable for the Chinese population. Therefore, we conducted a similar study using the Chinese Glioma Genome Atlas (CGGA) database, which is based on a Chinese population.

Accordingly, in this study, WGCNA was used to determine the association between functional gene clusters and clinicopathological parameters through the network construction of co-expressing genes based on the similarities in gene expression as well as analogous functions among all of the genes from collected samples of glioma patients, revealing the responsible genes within the most significant module in the network. Moreover, functional enrichment analysis of these identified genes also was carried out within the co-expression networks, thereby uncovering the underlying molecular mechanisms for the formation and progression of gliomas. Thus, the results of this study will provide a better understanding of the pathogenesis of gliomas and might offer a novel clinical treatment for Chinese glioma patients. We present the following article in accordance with the MDAR checklist (available at http:// dx.doi.org/10.21037/tcr-20-492).

\section{Methods}

\section{Data collection and pre-processing}

In accordance with the 2007 World Health Organization classification, the clinicopathological parameters of the eligible patients with gliomas along with their expression profiles (233 cases) were extracted from the CGGA database (http://www.cgga.org.cn). The following clinicopathological parameters of patients with histologically confirmed glioma, who had undergone a tumorectomy as well as follow-up treatment at Beijing Tiantan Hospital who established the CGGA database, were collected: age, gender, pathologic diagnosis, and outcome. Among them, the complete data of 53 patients (Table S1) who received chemotherapy and radiotherapy were selected for WGCNA to identify hub genes, which were further validated using the TCGA database (https://genome-cancer.ucsc.edu/) (9). The study was conducted in accordance with the Declaration of Helsinki (as revised in 2013). This study was approved by the review board of the local institution and the CGGA database of the Beijing Neurosurgical Institute at Capital Medical University. However, the requirement of written informed consent from the included patients with gliomas was waived. 


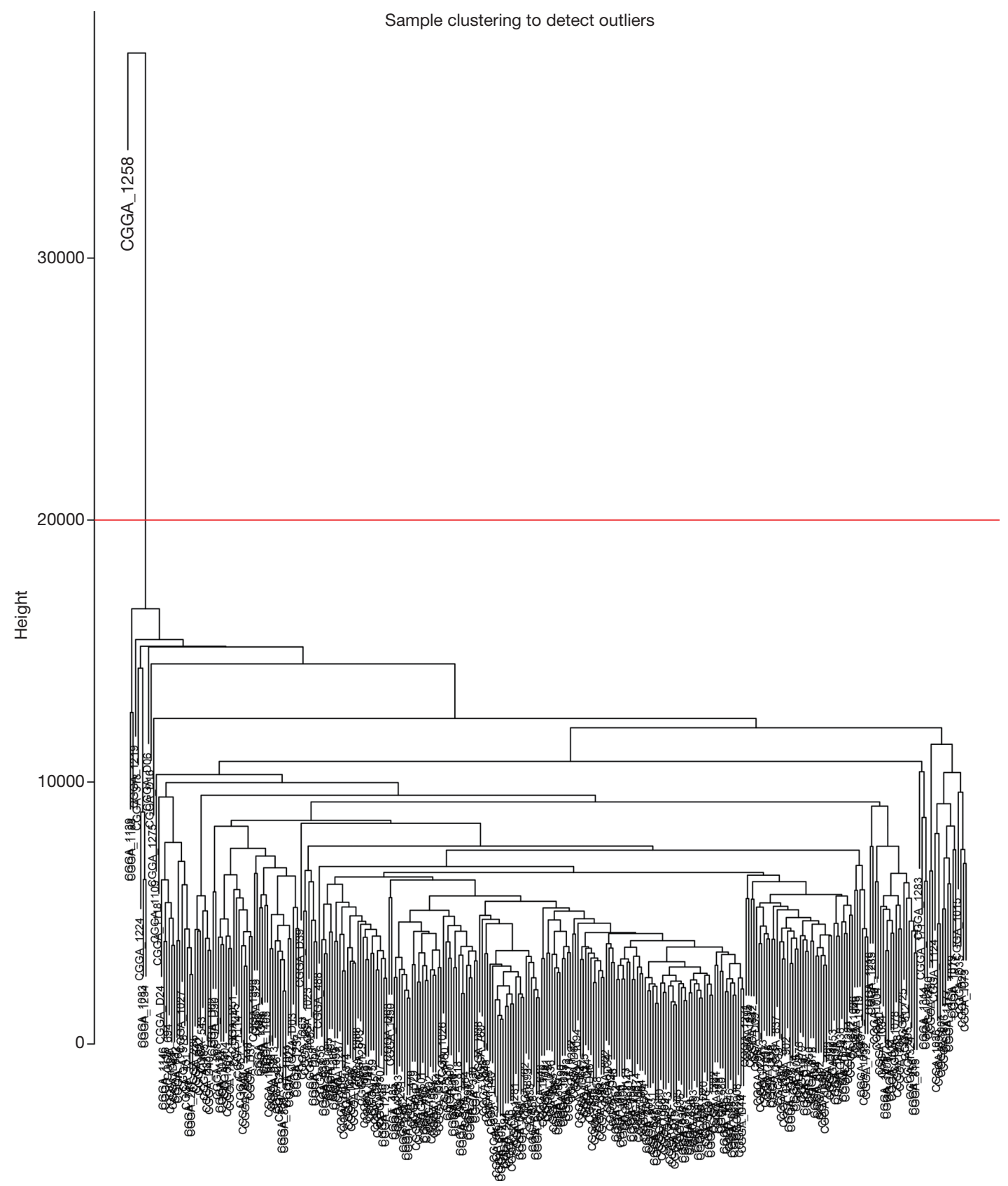

Figure 1 Clustering dendrogram of samples based on their Euclidean distance.

\section{Identification of clinically significant modules through WGCNA}

Following data pre-processing of the high-throughput mRNA sequencing data, the genes with increased genetic variance were collected for the systematic network construction of gene co-expression (Figure 1) by using the WGCNA (version 1.41; labs.genetics.ucla.edu/horvath/
coexpressionNetwork/Rpackages) algorithm (10). First, a scale-free topology was constructed for the network analysis with a proper $\beta$ power (the power of $\beta=9$ ) (Figure 2). Second, based on the topology overlap, hierarchical average linkage clustering was utilized to identify modules of interest, thus obtaining the specific gene modules associated with certain phenotypes, followed by visualization with plots within the 

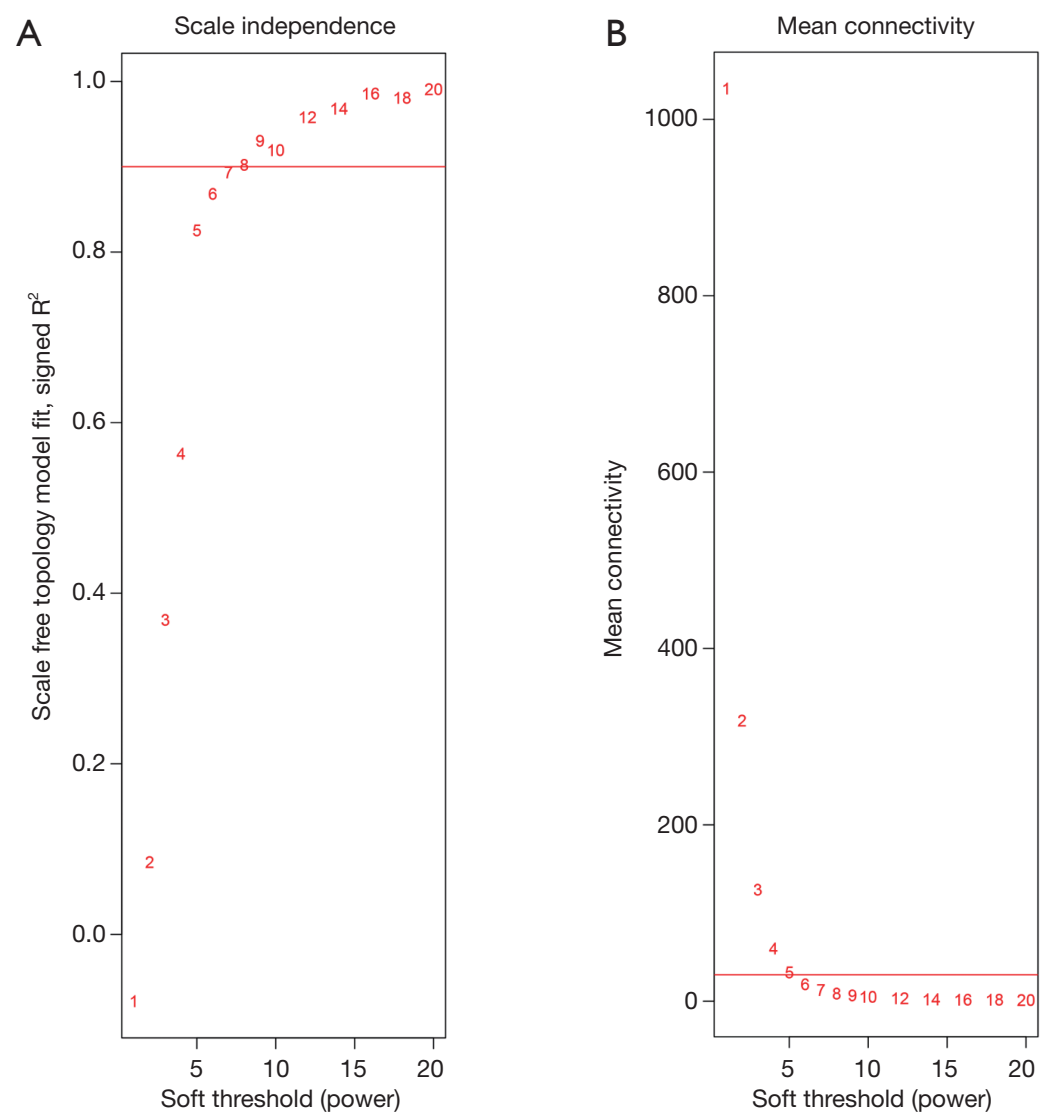

Figure 2 Determination of soft-thresholding power in WGCNA. (A) Analysis of the scale-free fit index for various soft-thresholding powers $(\beta)$. (B) Analysis of the mean connectivity for various soft-thresholding powers.

gene network. Finally, the association between the modules and the overall survival of the glioma patients receiving a combination of radiotherapy and chemotherapy was determined.

After the gene modules were identified, module significance was considered as the average gene significance among selected genes within one specific module (Figure 3). The module with the highest value of module significance compared to the other selected modules was regarded as a potential candidate that might be tightly associated with clinicopathological parameters (Figure 4). The gene module that was tightly associated with the overall survival of the glioma patients was then chosen for additional analysis. To further determine the potential signaling pathways of genes shown in the modules to be related with the clinicopathological parameters (Figure 5), the "clusterProfiler" package (11) in R was used to annotate and visualize the Kyoto Encyclopedia of Genes and Genomes (KEGG) pathways (12).

\section{Hub gene identification}

Hub genes, known as being extensively interconnected with other genes within the selected module, normally play essential roles in the network. Accordingly, modules of interest were chosen, and, in turn, critical hub genes were well defined by the connectivity of these modules based on the assessment of Pearson's correlation coefficients. Briefly, coefficients for evaluation of the association between module connectivity and hub genes should be greater than 0.8 , while coefficients for evaluation of the association between clinicopathological parameters and hub genes should be greater than 0.2. Moreover, the STRING database was also utilized for the construction of a model for protein-protein interactions (PPIs) when the confidence index of the related interaction was greater than 0.4 . Through the construction of PPI networks, 50 defined hub genes were subsequently ranked by using minimum multicollinearity methods (Figure 6). The common hub genes detected in either the 


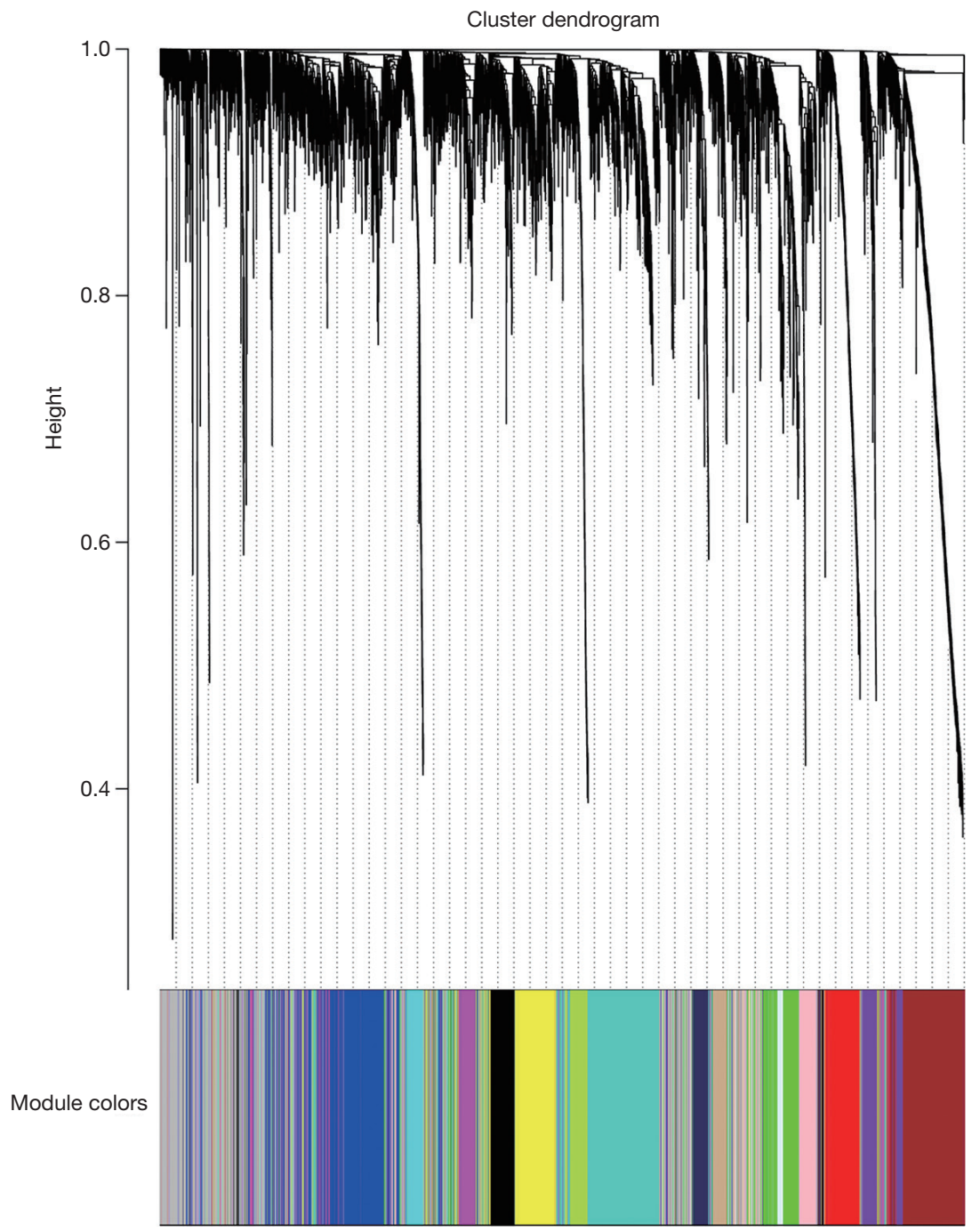

Figure 3 Clustering dendrogram of genes with dissimilarity based on the topological overlap, together with the assigned module colors.

co-expression network or the PPI network were considered as authentic hub genes for further validation.

\section{Evaluation of clinical efficacy}

The hub genes were first validated using the survival data of all glioma patients from the CGGA database and then further validated using the survival data of low-grade glioma patients from the TCGA database, especially for the assessment of overall survival rates to determine the effect of all hub genes on the prognosis of patients with glioma. The Oncomine database (http://www.oncomine.org) was also utilized for further validation of candidate gene expression (13).

\section{Statistical analysis}

Regarding the overall survival rate, patients with a complete survival time and a censor status were subdivided into two groups in accordance with the median expression level of each hub gene, namely, high group vs. low group (Figure 7). $\mathrm{R}$ software was used for the log-rank test as well as KaplanMeier survival analysis (14).

\section{Results}

\section{Identification of essential modules through WGCNA}

Following WGCNA of the mRNA co-expression networks, 18 modules were identified and labeled with distinct colors 


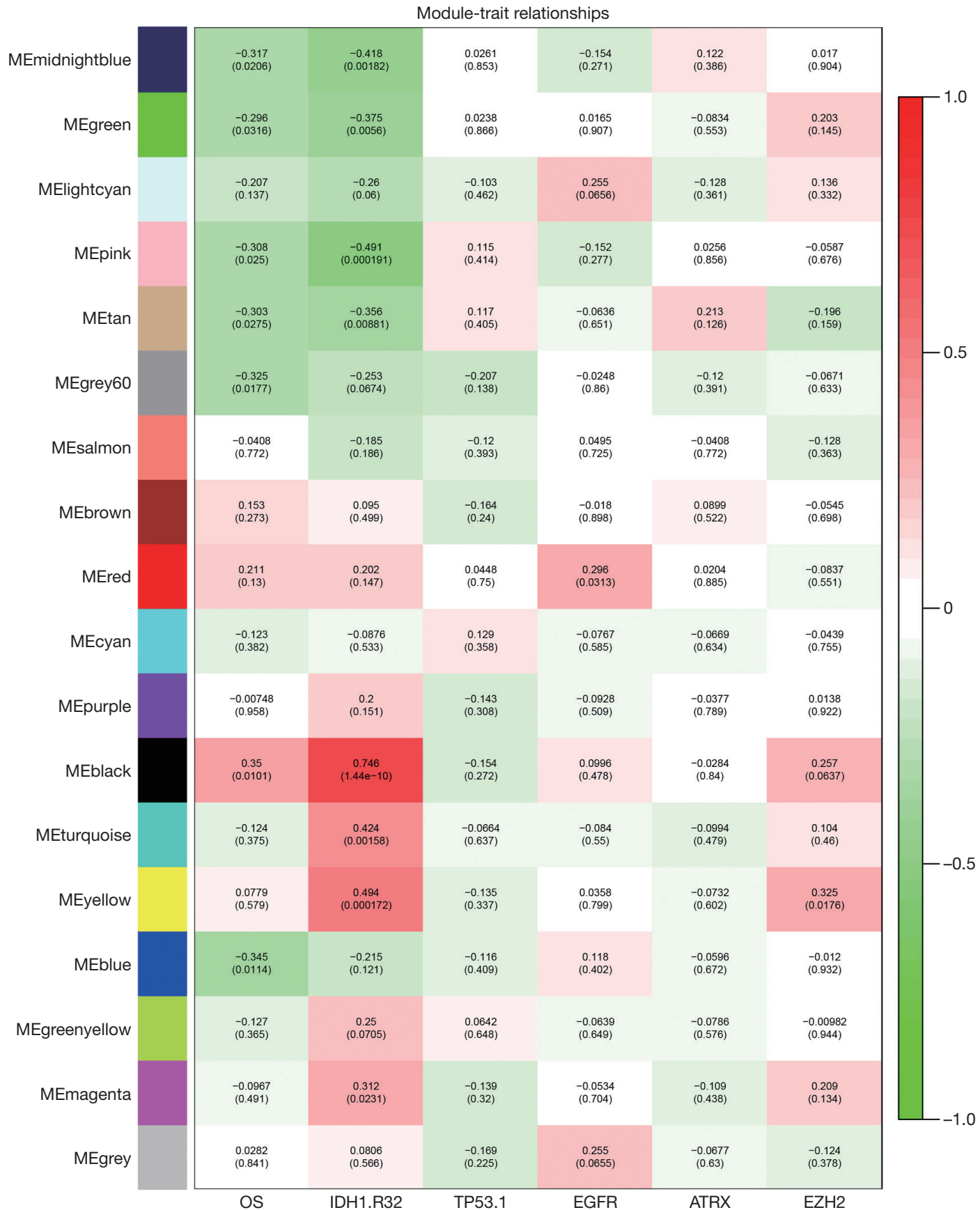

Figure 4 Module-trait associations were evaluated by correlations between module eigengenes (MEs) and clinical traits. Each row corresponds to a ME, while each column corresponds to a trait. Each cell contains the corresponding correlation (first line) and $\mathrm{P}$ value (second line). The figure is color-coded by correlation according to the color legend. The black module positively correlates to overall survival (OS) $(\mathrm{P}<0.05)$.

from the mRNA network. Among them, the black module, showing the most significant correlation with the clinical prognosis, was regarded as being the best prognostic indicator for tumor prognosis by using the correlation analysis for the sequencing results of 233 gliomas (Figures 3,4). Through computation with the KEGG 


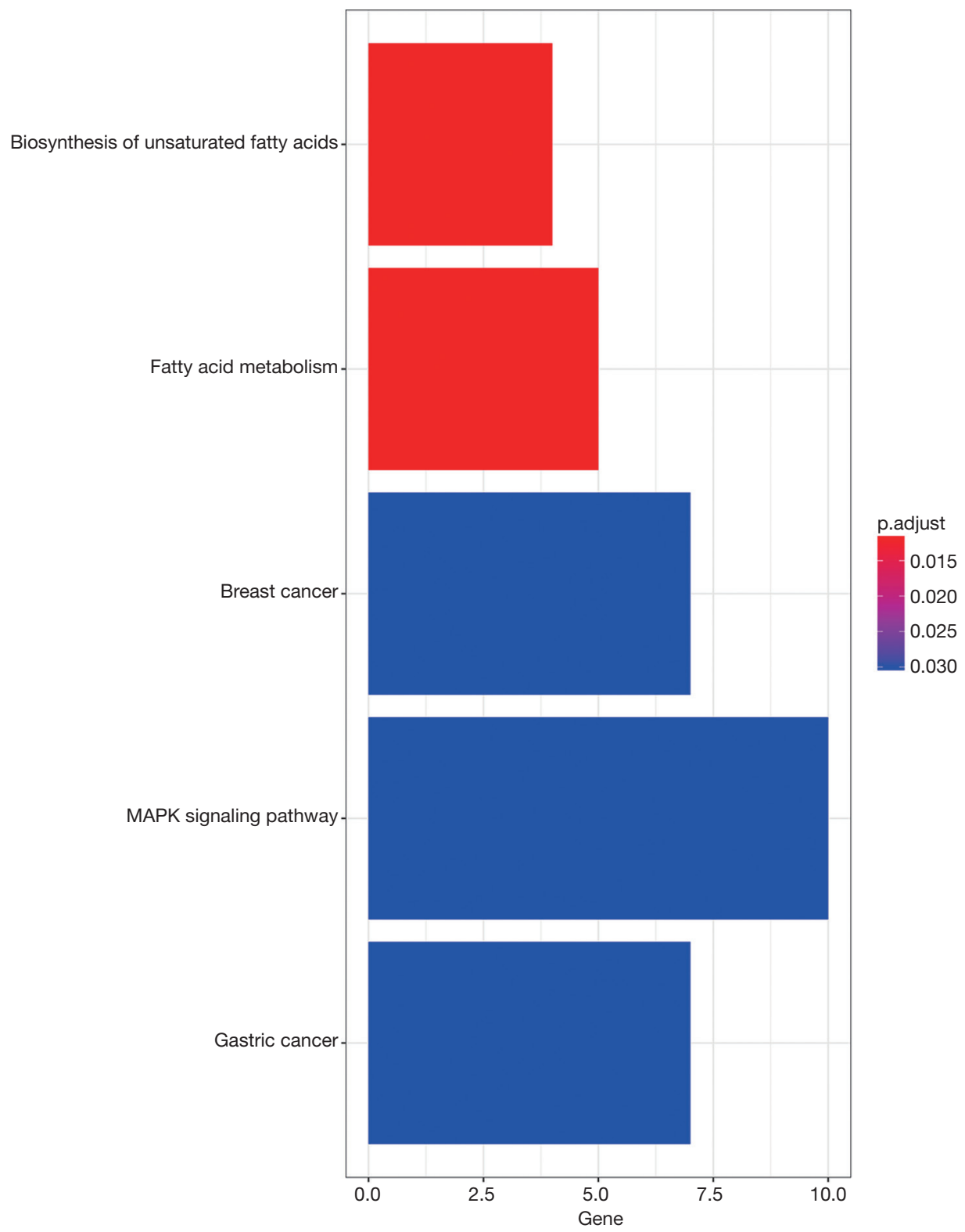

Figure 5 The most significantly enriched KEGG pathways of genes in the black module.

pathway database, functional enrichment analysis further revealed quite a few signaling pathways, such as the MAPK signaling pathway, underlying not only the formation and progression of breast/gastric cancer but also many vital biological processes, such as the biosynthesis of unsaturated fatty acids as well as fatty acid metabolism, suggesting that these correlated signaling pathways and biological processes potentially play indispensable roles in the formation/ progression of gliomas (Figure 5).

\section{Identification and validation of bub genes}

The 29 genes with the most extensive interconnections within the black module were defined as hub genes by the connectivity profile along with their association with the clinicopathological parameters, based on Pearson's correlation coefficient assessment (Figure 4). Besides, in accordance with the STRING database (https://string-db. org/), the PPI network was also constructed for further determination of all genes within the black module via 
Cytoscape (Figure 6). Thus, 50 defined hub genes were subsequently ranked by using minimum multicollinearity methods. Among them, five common hub genes (KCNB1, UST, SOX8, KLHL42, and HDAC4) that were found by analysis of either the co-expression or the PPI network were then accepted as authentic hub genes for further validation (15).

\section{Evaluation of clinical efficacy and survival analysis}

To further determine the correlation between the five hub genes and the related prognosis of glioma patients, survival curve analysis was performed by using Kaplan-Meier analysis. Prognostic cut-off criteria were set as the median values of the expression level of each hub gene. Through survival curve analysis, our results uncovered a significant difference in the overall survival rates of the glioma patients with distinct expression profiles of the selected hub genes, i.e., KCNB1, UST, SOX8, KLHL42, and HDAC4 (Figure 7), indicating that all these hub genes were potential prognostic biomarkers of glioma. Moreover, the KaplanMeier survival curve showed that a better discernibility was observed in data from the CGGA database (Figure 7).

\section{Discussion}

Glioma is known as the most lethal primary brain tumor, and glioma patients usually have a poor survival rate. In general, there are a variety of genetic alterations involved in glioma formation that primarily contribute to different responses to therapies. In this study, we aimed to identify novel biomarkers that offer better prognosis prediction in patients with glioma through analysis of a gene coexpression network. We hypothesized that the molecular mechanisms underlying any biological process require a close association of a co-expressed gene module sharing the same functional annotations. In this study, following comprehensive screening, 29 hub genes were specifically selected from the identified black module. Regarding the combined use of a co-expression network as well as a PPI network for further validation, five of them were considered as authentic hub genes (KCNB1, UST, SOX8, KLHL42, and HDAC4), suggesting that they are potentially promising indicators for assessment of vital biological processes as well as clinical outcomes. Furthermore, these five genes possessed a better prediction accuracy in Chinese patient samples from the CGGA database compared to samples from the TCGA database.

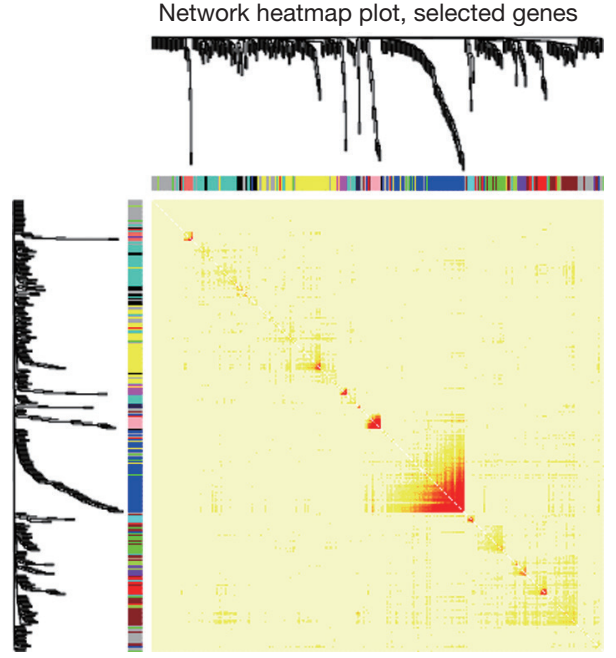

Figure 6 Heatmap for topological overlap in the gene network. In the heatmap, each row and column correspond to a gene; a light color denotes low topological overlap, and a progressively darker red denotes a higher topological overlap. Darker squares along the diagonal correspond to modules. The gene dendrogram and module assignment are shown along the left and top.

Specifically, our results showed that there was an apparent correlation of the identified black module with IDH mutations, which are widely accepted as being a distinct proneural genetic signature manifesting the glioma-CpG island methylator phenotype. Previous reports have revealed that IDH is not only associated with glioma formation/ progression but also with its recurrence and secondary glioblastomas (16); hence, it is a potential novel therapeutic target for the treatment of IDH-mutant gliomas (17).

On the other hand, accumulating evidence has revealed the involvement of some of these identified hub genes in various vital processes. For instance, there was an apparently enhanced amount of KCNB1 expression along with accumulated autophagic vacuoles during the early and late phases of apoptosis, possibly through an ERK/ MAPK-dependent signaling pathway (9). In addition, a heterogeneous pattern of Sox expression is generally regarded to be compatible with less-differentiated gliomas, as compared to their counterparts from the control group (18).

Moreover, the involvement of an epigenetic modification, such as histone modification, has been extensively investigated in tumorigenesis and chemotherapy/ radiotherapy resistance for more than a decade. Previous studies using immunohistochemical analysis have uncovered that an increased expression level of HDAC4 predicts a 

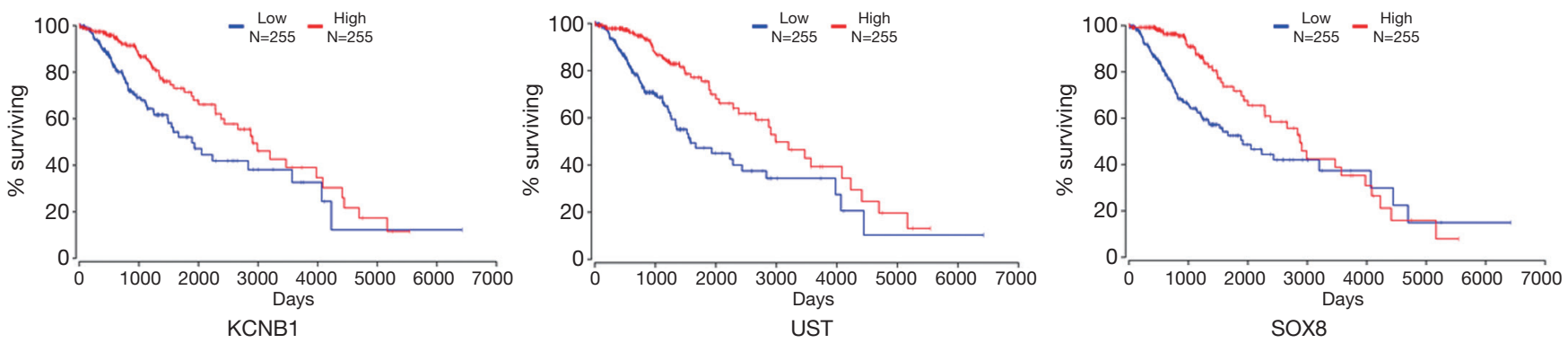

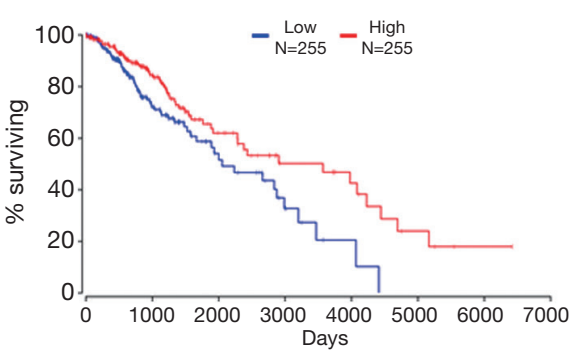

KLHL42

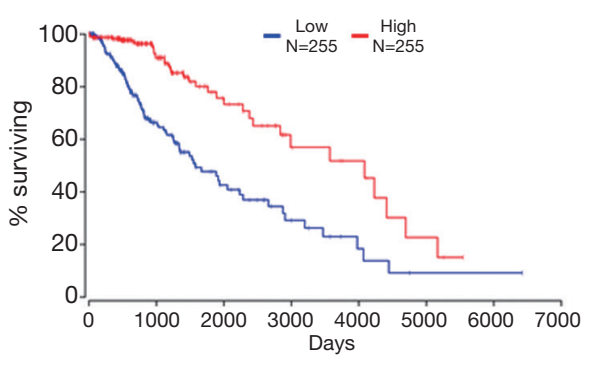

HDAC4

Figure 7 Kaplan-Meier plots for the genes KCNB1, UST, SOX8, KLHL42, and HDAC4 in glioma samples from the CGGA and TCGA databases.

poor overall survival for patients with glioma. However, HDAC4 is a highly abundant gene with a variety of vital biological functions in the brain. For example, HDAC4 is critical in the modulation of neuronal survival, thus strongly indicating that it at least partially contributes to therapeutic resistance and poor clinical outcomes in glioblastoma patients. Interestingly, significantly decreased expression of the HDAC gene has been reported to be closely associated with the etiology as well as tumor progression in grade-related astrocytoma (19). Likewise, the expression level of HDAC4 in combination with the methylation status of the promotor of the $\mathrm{O}_{6}$-methylguanine-DNA methyltransferase gene has been applied for the evaluation of glioblastoma for the prediction of its response to combined radiochemotherapy and prognosis after the therapy (20). Accordingly, there also have been a few reports on the essential role of HDAC4 mediating DNA-damage repair, which in turn reverses irradiation-induced DNA damage as well as stemness, resulting in the promotion of radioresistance in patients with glioblastoma $(21,22)$.

Although we used a similar method as $\mathrm{Xu}$ et al. (8), the five genes (KCNB1, UST, SOX8, KLHL42, and HDAC4) we identified were totally different from the four genes (OSMR, SOX21, MED10, and PTPRN) reported by $\mathrm{Xu}$ et al. (8). The difference in the data source was the main reason for the discrepancy. Furthermore, during gene identification, we focused on patients who received chemotherapy and radiotherapy. Since these genes were identified using cases from the CGGA database, it is not surprising that these genes can better predict cases from the CGGA database than those from the TCGA database.

Nevertheless, some limitations of this study should be pointed out. First, the data were extracted from the CGGA database for the determination and validation of co-expressed modules rather than from the collection of original data. Moreover, there were no molecular and/or biochemical assays for further examination and validation of these hypothetical associations and/or interactions, which likely include false-positive results.

In summary, through the network construction of coexpressed genes by using WGCNA, we identified as well as validated a few critical hub genes that were regarded to be closely associated with the prognosis of glioma in Chinese patients, including KCNB1, UST, SOX8, KLHDC5, and HDAC4. Hence, these hub genes are potentially of great clinical significance and could possibly lead to promising approaches for personalized tumor therapy.

\section{Acknowledgments}

We thank Medjaden Bioscience for help with proofreading this article. 
Funding: None.

\section{Footnote}

Reporting Checklist: The authors have completed the MDAR checklist. Available at http://dx.doi.org/10.21037/tcr-20492

Conflicts of Interest: All authors have completed the ICMJE uniform disclosure form (available at http://dx.doi. org/10.21037/tcr-20-492). The authors have no conflicts of interest to declare.

Ethical Statement: The authors are accountable for all aspects of the work in ensuring that questions related to the accuracy or integrity of any part of the work are appropriately investigated and resolved. The study was conducted in accordance with the Declaration of Helsinki (as revised in 2013). This study was approved by the review board of the local institution and the CGGA database of the Beijing Neurosurgical Institute at Capital Medical University. However, the requirement of written informed consent from the included patients with gliomas was waived.

Open Access Statement: This is an Open Access article distributed in accordance with the Creative Commons Attribution-NonCommercial-NoDerivs 4.0 International License (CC BY-NC-ND 4.0), which permits the noncommercial replication and distribution of the article with the strict proviso that no changes or edits are made and the original work is properly cited (including links to both the formal publication through the relevant DOI and the license). See: https://creativecommons.org/licenses/by-nc-nd/4.0/.

\section{References}

1. Bo L, Wei B, Li C, et al. Identification of potential key genes associated with glioblastoma based on the gene expression profile. Oncol Lett 2017;14:2045-52.

2. Sanai N, Berger MS. Recent surgical management of gliomas. Adv Exp Med Biol 2012;746:12-25.

3. Senders JT, Harary M, Stopa BM, et al. Information-Based Medicine in Glioma Patients: A Clinical Perspective. Comput Math Methods Med 2018;2018:8572058.

4. Kong Z, Yan C, Zhu R, et al. Imaging biomarkers guided anti-angiogenic therapy for malignant gliomas. Neuroimage Clin 2018;20:51-60.

5. Ludwig K, Kornblum HI. Molecular markers in glioma. J
Neurooncol 2017;134:505-12.

6. Picca A, Berzero G, Di Stefano AL, et al. The clinical use of IDH1 and IDH2 mutations in gliomas. Expert Rev Mol Diagn 2018;18:1041-51.

7. Qian $M$, Chen Z, Wang S, et al. PLEKHG5 is a novel prognostic biomarker in glioma patients. Int J Clin Oncol 2019;24:1350-8.

8. Xu P, Yang J, Liu J, et al. Identification of glioblastoma gene prognosis modules based on weighted gene coexpression network analysis. BMC Med Genomics 2018;11:96.

9. Wang HY, Wang W, Liu YW, et al. Role of KCNB1 in the prognosis of gliomas and autophagy modulation. Sci Rep 2017;7:14.

10. Langfelder P, Horvath S. WGCNA: an R package for weighted correlation network analysis. BMC Bioinformatics 2008;9:559.

11. Yu G, Wang LG, Han Y, et al. clusterProfiler: an R package for comparing biological themes among gene clusters. OMICS 2012;16:284-7.

12. Chen J, Wang X, Hu B, et al. Candidate genes in gastric cancer identified by constructing a weighted gene coexpression network. PeerJ 2018;6:e4692.

13. Chen L, Yuan L, Qian K, et al. Identification of Biomarkers Associated With Pathological Stage and Prognosis of Clear Cell Renal Cell Carcinoma by Coexpression Network Analysis. Front Physiol 2018;9:399.

14. Goel MK, Khanna P, Kishore J. Understanding survival analysis: Kaplan-Meier estimate. Int J Ayurveda Res 2010;1:274-8.

15. Chen L, Yuan L, Wang Y, et al. Co-expression network analysis identified FCER1G in association with progression and prognosis in human clear cell renal cell carcinoma. Int J Biol Sci 2017;13:1361-72.

16. Olar A, Wani KM, Alfaro-Munoz KD, et al. IDH mutation status and role of WHO grade and mitotic index in overall survival in grade II-III diffuse gliomas. Acta Neuropathol 2015;129:585-96.

17. Turkalp Z, Karamchandani J, Das S. IDH mutation in glioma: new insights and promises for the future. JAMA Neurol 2014;71:1319-25.

18. Schlierf B, Friedrich RP, Roerig P, et al. Expression of SoxE and SoxD genes in human gliomas. Neuropathol Appl Neurobiol 2007;33:621-30.

19. Gomes MV, Borges KS, Moreno DA, et al. Abnormal methylation of histone deacetylase genes: implications on etiology and epigenetic therapy of astrocytomas. Anticancer Res 2011;31:1337-43. 
20. Cheng W, Li M, Cai J, et al. HDAC4, a prognostic and chromosomal instability marker, refines the predictive value of MGMT promoter methylation. J Neurooncol 2015;122:303-12.

21. Marampon F, Megiorni F, Camero S, et al. HDAC4 and HDAC6 sustain DNA double strand break repair and stem-like phenotype by promoting radioresistance in glioblastoma cells. Cancer Lett 2017;397:1-11.

22. Sferra R, Pompili S, Festuccia C, et al. The possible prognostic role of histone deacetylase and transforming growth factor beta/Smad signaling in high grade gliomas treated by radio-chemotherapy: a preliminary immunohistochemical study. Eur J Histochem 2017;61:2732.
Cite this article as: Jiang YW, Wang R, Zhuang YD, Chen $\mathrm{CM}$. Identification and validation of potential novel prognostic biomarkers for patients with glioma based on a gene coexpression network. Transl Cancer Res 2020;9(10):6444-6454. doi: $10.21037 /$ tcr-20-492 
Table S1 The complete data of 55 patients for WGCNA

\begin{tabular}{|c|c|c|c|c|c|c|c|c|c|c|}
\hline CGGA_ID & os & Status & Radio & Chemo & IDH1.R32 & IDH2.R172 & TP53.1 & EGFR & ATRX & $\mathrm{EZH} 2$ \\
\hline CGGA_1006 & 254 & 1 & 1 & 1 & 0 & 0 & 0 & 1 & 0 & 0 \\
\hline CGGA_1007 & 345 & 1 & 1 & 1 & 0 & 0 & 1 & 0 & 1 & 0 \\
\hline CGGA_1035 & 567 & 1 & 1 & 1 & 0 & 0 & 1 & 0 & 1 & 0 \\
\hline CGGA_1045 & 347 & 1 & 1 & 1 & 0 & 0 & 1 & 0 & 0 & 0 \\
\hline CGGA_1072 & 84 & 1 & 1 & 1 & 0 & 0 & 0 & 0 & 0 & 0 \\
\hline CGGA_1081 & 216 & 1 & 1 & 1 & 0 & 0 & 1 & 0 & 0 & 0 \\
\hline CGGA_1145 & 239 & 1 & 1 & 1 & 0 & 0 & 0 & 1 & 0 & 0 \\
\hline CGGA_1177 & 449 & 1 & 1 & 1 & 0 & 0 & 1 & 0 & 0 & 0 \\
\hline CGGA_1224 & 271 & 1 & 1 & 1 & 0 & 0 & 1 & 0 & 0 & 1 \\
\hline CGGA_1237 & 249 & 1 & 1 & 1 & 0 & 0 & 1 & 0 & 0 & 0 \\
\hline CGGA_309 & 153 & 1 & 1 & 1 & 0 & 0 & 0 & 0 & 0 & 0 \\
\hline CGGA_330 & 1,121 & 1 & 1 & 1 & 0 & 0 & 0 & 0 & 0 & 0 \\
\hline CGGA_426 & 1,560 & 1 & 1 & 1 & 0 & 0 & 1 & 0 & 0 & 0 \\
\hline CGGA_448 & 354 & 1 & 1 & 1 & 0 & 0 & 0 & 1 & 0 & 1 \\
\hline CGGA_483 & 271 & 1 & 1 & 1 & 0 & 0 & 0 & 0 & 0 & 0 \\
\hline CGGA_488 & 435 & 1 & 1 & 1 & 0 & 0 & 1 & 0 & 0 & 0 \\
\hline CGGA_564 & 679 & 1 & 1 & 1 & 0 & 0 & 0 & 0 & 0 & 0 \\
\hline CGGA_578 & 376 & 1 & 1 & 1 & 0 & 0 & 0 & 0 & 0 & 0 \\
\hline CGGA_604 & 381 & 1 & 1 & 1 & 0 & 0 & 0 & 0 & 0 & 0 \\
\hline CGGA_616 & 952 & 1 & 1 & 1 & 0 & 0 & 1 & 0 & 0 & 0 \\
\hline CGGA_643 & 331 & 1 & 1 & 1 & 0 & 0 & 0 & 0 & 0 & 0 \\
\hline CGGA_658 & 372 & 1 & 1 & 1 & 0 & 0 & 1 & 0 & 0 & 0 \\
\hline CGGA_669 & 607 & 1 & 1 & 1 & 0 & 0 & 0 & 0 & 0 & 0 \\
\hline CGGA_676 & 376 & 1 & 1 & 1 & 0 & 0 & 1 & 0 & 0 & 0 \\
\hline CGGA_678 & 386 & 1 & 1 & 1 & 0 & 0 & 1 & 0 & 0 & 0 \\
\hline CGGA_727 & 1,023 & 1 & 1 & 1 & 0 & 0 & 1 & 1 & 0 & 0 \\
\hline CGGA_796 & 720 & 1 & 1 & 1 & 0 & 0 & 1 & 1 & 0 & 0 \\
\hline CGGA_802 & 681 & 1 & 1 & 1 & 0 & 0 & 1 & 0 & 0 & 0 \\
\hline CGGA_808 & 309 & 1 & 1 & 1 & 0 & 0 & 1 & 1 & 0 & 0 \\
\hline CGGA_837 & 432 & 1 & 1 & 1 & 0 & 0 & 1 & 1 & 0 & 0 \\
\hline CGGA_842 & 234 & 1 & 1 & 1 & 0 & 0 & 1 & 0 & 0 & 0 \\
\hline CGGA_D02 & 484 & 1 & 1 & 1 & 0 & 0 & 0 & 0 & 0 & 0 \\
\hline CGGA_D03 & 423 & 1 & 1 & 1 & 0 & 0 & 0 & 1 & 0 & 0 \\
\hline CGGA_D11 & 827 & 1 & 1 & 1 & 0 & 0 & 1 & 0 & 0 & 1 \\
\hline CGGA_D37 & 965 & 1 & 1 & 1 & 0 & 0 & 1 & 0 & 0 & 0 \\
\hline CGGA_D57 & 766 & 1 & 1 & 1 & 0 & 0 & 1 & 0 & 0 & 0 \\
\hline CGGA_D62 & 514 & 1 & 1 & 1 & 0 & 0 & 0 & 0 & 0 & 0 \\
\hline CGGA_1215 & 29 & 1 & 1 & 1 & 1 & 0 & 1 & 1 & 0 & 0 \\
\hline CGGA_300 & 2,199 & 1 & 1 & 1 & 1 & 0 & 1 & 0 & 1 & 0 \\
\hline CGGA_491 & 1,074 & 1 & 1 & 1 & 1 & 0 & 1 & 1 & 1 & 1 \\
\hline CGGA_545 & 555 & 1 & 1 & 1 & 1 & 0 & 1 & 1 & 0 & 0 \\
\hline CGGA_591 & 1,654 & 1 & 1 & 1 & 1 & 0 & 0 & 1 & 0 & 0 \\
\hline CGGA_632 & 572 & 1 & 1 & 1 & 1 & 0 & 0 & 1 & 0 & 0 \\
\hline CGGA_694 & 1,194 & 1 & 1 & 1 & 1 & 0 & 0 & 0 & 0 & 0 \\
\hline CGGA_747 & 970 & 1 & 1 & 1 & 1 & 0 & 0 & 0 & 0 & 1 \\
\hline CGGA_757 & 1,047 & 1 & 1 & 1 & 1 & 0 & 1 & 0 & 0 & 0 \\
\hline CGGA_884 & 1,131 & 1 & 1 & 1 & 1 & 0 & 1 & 0 & 0 & 0 \\
\hline CGGA_D47 & 1,048 & 1 & 1 & 1 & 1 & 0 & 0 & 0 & 0 & 1 \\
\hline CGGA_J023 & 1,028 & 1 & 1 & 1 & 1 & 0 & 1 & 0 & 0 & 0 \\
\hline CGGA_1008 & 315 & 1 & 1 & 1 & 0 & 0 & 1 & 0 & 0 & 0 \\
\hline CGGA_1119 & 436 & 1 & 1 & 1 & 1 & 0 & 1 & 0 & 0 & 0 \\
\hline CGGA_247 & 609 & 1 & 1 & 1 & 1 & 0 & 0 & 0 & 0 & 1 \\
\hline CGGA_759 & 1,263 & 1 & 1 & 1 & 1 & 0 & 1 & 1 & 0 & 0 \\
\hline
\end{tabular}

